Y.-H. Chu, N.B. Suntzeff, J.E. Hesser, and D.A. Bohlender, eds.

\title{
The Metallicity and Dust Content of HVC287+22+240: Evidence for a Magellanic Clouds Origin
}

\author{
Bart Wakker, Blair D. Savage \\ Dept. of Astronomy, Univ. of Wisconsin, Madison, WI 53706, USA \\ Limin Lu, Wallace L.W. Sargent \\ California Institute of Technology, 105-24, Pasadena, CA 91125, USA
}

\author{
Kenneth R. Sembach \\ Department of Physics and Astronomy, Johns Hopkins University, \\ Baltimore, MD 21218, USA
}

\section{Tom A. Oosterloo \\ CNR, Istituto di Fisica Cosmica, Milan, Italy}

We present evidence that the properties of the high-velocity cloud catalogued as \#187 by Wakker \& van Woerden (1991) (HVC287+22+240) can be explained if it is part of a leading arm counterpart of the Magellanic Stream, and we use this to argue that the HVC originated in the Magellanic Clouds after a tidal interaction.

The sulphur and iron abundance were measured using GHRS spectra of NGC3783, a background Seyfert galaxy. These data were fully described by Lu et al (1998). An accurate reference HI column density was found by combining data taken with the Australia Telescope Compact Array with data from the Green Bank $140 \mathrm{ft}$ telescope. The particle distributions predicted by the tidal model of Gardiner \& Noguchi (1996) are compared with those of a subsample of the high-velocity clouds.

We find that $(\mathrm{S} / \mathrm{H})=0.25 \pm 0.07$ solar in the $\mathrm{HVC}$, providing the first accurate measurement of the chemical enrichment level in an HVC. Note that this is similar to the interstellar abundance of $\mathrm{S}$ in the Magellanic Clouds: $(\mathrm{S} / \mathrm{H})=0.27$ solar in the LMC, and 0.21 solar in the SMC (Russel \& Dopita 1992).

We find that $(\mathrm{Fe} / \mathrm{H})=0.13 \pm 0.04$ solar in the HVC. In diffuse disk gas subsolar ratios indicate that most of the iron is locked into dust $((\mathrm{Fe} / \mathrm{S}) \sim 0.005$ solar in cool gas, $(\mathrm{Fe} / \mathrm{S}) \sim 0.25$ solar in warm gas; Savage \& Sembach 1996). The ratio in the HVC implies that 7 out of $8 \mathrm{Fe}$ atoms are in dust (if $\mathrm{Fe} / \mathrm{S}$ is intrinsically solar, as is the case in the SMC). If the intrinsic ratio were that in metal-poor halo stars $(\sim 0.4$, Wheeler, Sneden \& Truran 1989), 2 out of $3 \mathrm{Fe}$ atoms are in dust. The $\mathrm{Fe} / \mathrm{S}$ ratio is similar to the interstellar ratio in the SMC, which is found by combining (Fe/Zn) 0.25 (Welty et al. 1997; Roth \& Blades 1997), with (Zn/S) 1 (Russel \& Dopita 1992).

HVC287+22+240 was interpreted as an extra-galactic cloud by West et al. (1985) on the basis of its low value of $(\mathrm{Ca} / \mathrm{H})(0.014 \pm 0.006$ solar $)$; however, this ratio suffers from an unknown ionization and depletion correction. Blitz 
et al. (1996) argued that many HVCs, including HVC287 $+22+240$, are best explained as "remnants of Local Group formation", and they used the subsolar $\mathrm{S}$ abundance as supporting evidence, although our observed $\mathrm{S}$ abundance would be rather high for relatively unprocessed gas.

Gardiner \& Noguchi (1996) presented an N-body simulation of the Magellanic system, modeling the Milky Way and the LMC as fixed potentials, but the SMC as a self-gravitating collection of particles. The model produces the Magellanic Stream, Magellanic Bridge and other features; especially noteworthy for our purposes is the prediction for the properties of the leading tidal arm. When we compare these predictions with the sky and velocity distribution of some HVCs, we find that they have similar global properties, although there are differences in the details.

We argue that the metallicity and depletion information for the HVC, coupled with its velocity and position on the sky, strongly suggest that the HVC (and by implication also the Magellanic Stream proper), originated from the Magellanic Clouds as the result of a tidal interaction between the LMC and the SMC. The alternative model for the origin of the Magellanic Stream is that it is the result of ram-pressure stripping by a tenuous galactic corona (Moore \& Davis 1994), but in this model the presence of gas with Magellanic abundances ahead of the Stream cannot be explained.

\section{References}

Blitz, L., Spergel, D., Teuben, P., Hartmann, D., \& Burton, W.B. 1996, BAAS, $189,61.01$

Gardiner, L.T., \& Noguchi, M. 1996, MNRAS, 278, 191

Lu, L., Savage, B.D., Sembach, K.R., Wakker, B.P., Sargent, W.W.L., \& Oosterloo, T.A. 1997, AJ, 115, 162

Moore, B., \& Davis, M. 1994, MNRAS, 270, 209

Roth, K.C, \& Blades, J.C. 1996, ApJ, 474, 95

Russel, S.C., \& Dopita, M.A. 1992, ApJ, 384, 508

Savage, B.D., \& Sembach, K.R. 1996, ARA\&A, 34, 279

Wakker, B.P., \& van Woerden, H. 1991, A\&A, 250, 509

Welty, D.E., Lauroesch, J.T., Blades, J.C., Hobbs, L.M., \& York, D.G. 1998, ApJ, 429, 627

West, K.A., Pettini ,M., Penston, M.V., Blades, J.C., \& Morton, D.C. 1985, MNRAS, 215, 481

Wheeler, J.C., Sneden, C., \& Truran, J.W. 1989, ARA\&A, 27, 279 